FERMILAB-PUB-88/151-A

0

NSF-ITP-88-148

October 1988

\title{
令 COSMOLOGY OF BIASED DISCRETE SYMMETRY BREAKING
}

Graciela B. Gelmini ${ }^{1) *}$, Marcelo Gleiser ${ }^{2)}$ and Edward W. Kolb ${ }^{3)}$

1) International Center for Theoretical Physics, and International School for Advanced Studies

Box 586, 34100 Trieste, Italy

2) Institute for Theoretical Physics

University of California

Santa Barbara, CA 99106

3) NASA/Fermilab Astrophysics Center

Fermi National Accelerator Laboratiory

Box 500, Batavia, IL 60510

We study cosmological consequences of spontaneous breaking of an approximate discrete symmetry. The breaking leads to formation of proto-domains of false and true vacuum separated by domain walls of thickness determined by the mass scale of the model. The cosmological evolution of the walls is extremely sensitive to the magnitude of the biasing; several scenarios are possible, depending on the interplay between the surface tension on the walls and the volume pressure from the biasing. Walls may disappear almost immediately after they form, or may live long enough to dominate the energy density of the Universe

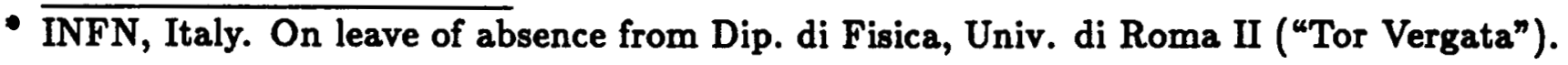


and cause power-law inflation. We obtain limits on the biasing that characterize each possible scenario.

ORIGINAL PAGE IS

OF POOR QUALITY 


\section{Introduction}

It has been known for some time that spontaneous breaking of a discrete symmetry leads to formation of surface-like topological defects called domain walls. ${ }^{1}$ These two-dimensional structures are stable if the vacuum manifold determined by the interaction potential is topologically non-trivial in the sense that it is defined by disconnected points related to discrete, degenerate ground states of the theory. Once the field settles into one of the possible ground states of the theory (i.e., a point in the vacuum manifold) it cannot continuously be transformed into another point in the vacuum manifold. The transition region between degenerate ground states is the domain wall. In the rest of this section we very briefly review these ideas.

The appearance of walls in a theory with discrete symmetry breaking can be very easily understood with a simple model: Consider a real scalar field $\sigma$ with Lagrangian density

$$
\mathcal{L}=\frac{1}{2}\left(\partial_{\mu} \sigma\right)^{2}-\frac{\lambda}{4}\left(\sigma^{2}-\sigma_{0}^{2}\right)^{2} .
$$

The $Z_{2}$ symmetry of the Lagrangian is broken when $\sigma$ obtains a vacuum expectation value $\sigma=+\sigma_{0}$ or $\sigma=-\sigma_{0}$. The transition region between the two possible vacuum values for $\sigma$ defines the domain wall.

Domain walls can be described by the solutions to the equation of motion obtained from the Lagrangian of Eq. (1),

$$
\eta^{\mu \nu} \sigma_{, \mu, \nu}+\lambda \sigma\left(\sigma^{2}-\sigma_{0}^{2}\right)=0 .
$$

Imagine an infinite wall in the $y-z$ plane at $x=0$. The solution with boundary conditions $\sigma=-\sigma_{0}$ at $x=-\infty$, and $\sigma=+\sigma_{0}$ at $x=+\infty$, is simply

$$
\sigma(x)=\sigma_{0} \tanh (x / \Delta)
$$

with $\Delta \equiv(\lambda / 2)^{1 / 2} \sigma_{0}^{-1}$ defined as the "thickness" of the wall. Balance between the potential energy that tends to make the wall thinner, and the gradient term that tends to make the 
ORIGINAL PACE IS

OF POOR QUALITY

wall thicker, gives rise to the finite thickness of the wall separating regions of different vacua.

The stress tensor for the wall, $T_{\mu \nu}=-\partial_{\mu} \sigma \partial_{\nu} \sigma-\mathcal{L} \eta_{\mu \nu}$, is

$$
T_{\nu}^{\mu}=\frac{\lambda}{2} \sigma_{0}^{4} \cosh ^{-4}(x / \Delta) \operatorname{diag}(1,0,1,1) .
$$

From $T^{\mu}{ }_{\nu}$ we can define the surface tension of the wall, i.e. the energy per unit area in the rest frame of the wall,

$$
\mu \equiv \int T_{0}^{0} d x=\int\left[\frac{1}{2}(\nabla \sigma)^{2}+\frac{\lambda}{4}\left(\sigma^{2}-\sigma_{0}^{2}\right)^{2}\right] d x,
$$

which is given by

$$
\mu=\frac{2 \sqrt{2}}{3} \lambda^{1 / 2} \sigma_{0}^{3}
$$

Since T. D. Lee's model for CP-violation of the early seventies, ${ }^{2}$ there have been many models where the presence of a real scalar field with quartic self-interaction leads to the spontaneous breaking of a discrete symmetry. For example, axion models which have been proposed as a solution to the strong CP problem in QCD have a spontaneously broken $Z_{N}$ that generates discrete degenerate vacua. ${ }^{3}$ We mention these two models because they were particularly important to the development of research on the role of topological defects in cosmology.

The first effort to understand the cosmological effects of spontaneous breaking of a discrete symmetry was the work by Zel'dovich, Kobzarev, and Okun. ${ }^{4}$ They outlined most of the details for the cosmological evolution of a Universe filled with walls in the case of degenerate vacua. Their conclusions were quite dramatic: The wall contribution to the energy density of the Universe will quickly overpower the radiation contribution, causing a period of power-law inflation (of course, their work pre-dated the proposal of inflation) with the scale factor going as $R(t) \sim t^{2}$. An expansion rate that fast would leave less time for galaxy formation and change the production rates during nucleosynthesis. 
Also, the presence of a wall in the observable Universe would cause distortions in the cosmic microwave background that would violate the present limits on its homogeneity and isotropy, unless the wall's gravitational redshift is safely below the detectable limit of $\delta T / T \leq \mathcal{O}\left(10^{-4}\right)$. These limits imply an upper bound on the surface tension $\mu \leq \mathcal{O}\left(10^{-1}\right)$ $\mathrm{g} \mathrm{cm}^{-2}$ or $\lambda^{1 / 6} \sigma_{0} \leq \mathcal{O}\left(10^{-2}\right) \mathrm{Gev}^{4}$ Thus, unless these limits could be satisfied (or somehow walls disappear early enough in the cosmological expansion), models with discrete symmetry breaking are ruled out by cosmology.

As the above limit on the scale for symmetry breaking seems very restrictive from the particle physics side, attention turned to the other way to accommodate walls, namely to have them exist for only a brief period. In fact, Zel'dovich, Kobzarev and Okun suggested that if the discrete symmetry was not exact, the energy difference between the two vacua would cause the false vacuum regions to disappear, possibly before the walls could dominate the energy density of the Universe. This same idea was mentioned in the works of Kibble, ${ }^{5}$ Vilenkin, ${ }^{6}$ and Sikivie. ${ }^{7} \mathrm{~A}$ small bias favouring one vacuum over the other (or others) can indeed make the walls go away. In Refs. (6-7) a lower bound for the asymmetry was obtained based upon the requirement that the walls should disappear before they dominate the cosmological evolution. Nevertheless, as we will point out in this work, the dynamics of the walls subject to both the usual tension force due to curvature and to the volume pressure due to the biasing is much richer than it has formerly been appreciated. The two forces will clearly compete with each other, with the resulting wall dynamics extremely sensitive to the magnitude of the bias. We will study in detail the cosmological consequences of the breaking of an approximate discrete symmetry (a "quasi-symmetry").

Different scenarios we consider may also be of relevance in the case of hybrid topological structures such as walls bounded by strings, ${ }^{1}$ or in the recently developed scenario for formation of non-topological solitons (hereafter NTSs). ${ }^{8}$ The case of NTSs is particularly interesting; the real scalar field that gives rise to the walls couples to a complex scalar 
field (or a fermion field) that carries a conserved global charge in such a way as possibly to allow for a small violation of the discrete symmetry (in fact it is demanded in the model of Ref. 8). In the false vacuum (the NTS interior) the complex scalar field is massless while in the true vacuum it is massive. The two regions are separated by a domain wall of thickness set by the mass of the real scalar field.

Due to the many possible situations of interest, we will not analyse any particular model. We will try, instead, to keep our results as general as possible. In Fig. 1 we show the general shape of the potential that we will consider. More details about the general model are given in Section 3.

The paper is organized as follows. In Section 2 we develop the kinematics of walls by considering a perfect gas of walls moving with an average velocity $w$ in a box of volume $V$. We will obtain a velocity-dependent equation of state for the wall gas. Next, by assuming that the energy density of the Universe is dominated by the wall gas, we will obtain the velocity-dependent law of cosmological expansion. In particular, we will show that the maximum wall velocity that results in power-law inflation is $w=1 / \sqrt{3}$, the sound speed in a relativistic gas. This section can be read quite independently from the rest of the paper. In Section 3 we will describe the formation of walls in a primordial phase transition in the presence of biasing. The dynamics of walls will be studied in Sections 4 and 5 , assuming, in section 4 , that the volume pressure acts before the tension force can stretch the walls to the horizon scale, and, in Section 5, assuming the biasing is small enough to allow the tension force to straighten walls to the horizon scale. In the second case, walls may dominate the energy density of the Universe, and cause a power-law inflation. Finally, in Section 6 we will review our results and discuss possible future directions for further work. 


\section{Kinematics of Walls and Evolution of a Wall-Dominated Universe}

In this section we obtain the equation of state for a perfect gas of walls moving with average velocity $w$ inside a box of volume $V \gg \Delta^{3}$, where $\Delta$ was defined in the introduction as the wall's thickness. The perfect gas assumption means that we will neglect possible dissipative effects that may come from interactions between walls.

Consider $N$ walls in the $y-z$ plane, moving in the $x$ direction with average velocity $w$. Each wall's position is described by the point $x^{i}$, with the index $i$ running from 1 to $N$. If there are many walls inside the box such that the average wall separation is much smaller than the linear dimensions of the box, we can write an average energy-momentum tensor for the $N$ walls as

$$
\left\langle T_{\mu \nu}\right\rangle=\frac{\int d x f(x) T_{\mu \nu}(x)}{\int d x f(x)},
$$

where $f(x)$ is the distribution function for the walls, $\int f(x) d x=N$. If $\langle L\rangle$ is the average wall separation, we can approximate $\left\langle T_{\mu \nu}\right\rangle$ as

$$
\left\langle T_{\mu \nu}\right\rangle \simeq \frac{1}{\langle L\rangle} \int T_{\mu \nu} d x \equiv \frac{1}{\langle L\rangle} S_{\mu \nu} .
$$

The tensor $S_{\mu \nu}$ can be understood as the average energy-momentum surface density of the walls. For example, $S_{00}=\mu$, where $\mu$ is the surface tension of the walls defined in Eq. (5). It should not be confused with the spacetime index $\mu$. Thus, the wall energy-density is given by $\rho_{W} \equiv\left\langle T_{00}\right\rangle=\mu /\langle L\rangle$.

The walls will be moving with average velocity $w$ in the $+\hat{x}$ direction with respect to an observer at rest with the box. Accordingly, the tangential components of $S_{\mu \nu}$ (i.e. $S_{22}$ and $S_{33}$ ) will not be affected by the walls' motion. ${ }^{4}$ The same cannot be said of the other components. Upon Lorentz transformation, $S_{\mu \nu}$ for an observer at rest is

$$
S_{\mu \nu}=\left(\begin{array}{cccc}
\gamma^{2} \mu & \gamma^{2} \mu w & 0 & 0 \\
\gamma^{2} \mu w & \gamma^{2} \mu w^{2} & 0 & 0 \\
0 & 0 & -\mu & 0 \\
0 & 0 & 0 & -\mu
\end{array}\right)
$$


where $\gamma \equiv\left(1-w^{2}\right)^{-1 / 2}$ as usual. Of course, there will be walls moving in the $-\hat{x}$ direction as well. Once we average the two directions, the off-diagonal terms disappear. In the most general case, the same procedure must be repeated for the $\pm \hat{y}$ and for the $\pm \hat{z}$ directions. The final result for the average energy-momentum tensor for a gas of walls moving with velocity $w$ with respect to an observer at rest is then

$$
\left\langle T_{\mu \nu}\right\rangle=\frac{\mu}{3\langle L\rangle}\left(\begin{array}{cccc}
3 \gamma^{2} & 0 & 0 & 0 \\
0 & \left(w^{2} \gamma^{2}-2\right) & 0 & 0 \\
0 & 0 & \left(w^{2} \gamma^{2}-2\right) & 0 \\
0 & 0 & 0 & \left(w^{2} \gamma^{2}-2\right)
\end{array}\right)
$$

Defining the energy density as $\rho_{W} \equiv\left\langle T_{00}\right\rangle$ and the pressure as $p_{W} \equiv\left\langle T_{i i}\right\rangle$, the equation of state for the wall gas becomes

$$
p_{W}=\frac{\left(w^{2} \gamma^{2}-2\right)}{3 \gamma^{2}} \rho_{W}
$$

We point out three cases of interest: For $w$ relativistic $(w \sim 1, \gamma \gg 1), p_{W}=$ $\rho_{W} / 3$, and the walls behave like a relativistic gas; for $w$ non-relativistic $(w \sim 0, \gamma \sim 1)$, $p_{W}=-2 \rho_{W} / 3$, which is the well-known result for static walls; and finally, for $w \simeq 0.82$ $\left(w^{2} \gamma^{2}=2\right), p_{W}=0$, and the walls behave as pressureless matter. Thus, for $w \geq 0.82$ the gas of walls will contribute positive pressure to the energy-momentum tensor.

Once we have the equation of state for the wall gas, it is natural to ask how the evolution of a Universe filled with walls scales with the velocity of the walls. For simplicity we take the flat Robertson-Walker metric as the spacetime metric, $d s^{2}=-d t^{2}+R(t)^{2}\left(d x^{2}+d y^{2}+d z^{2}\right)$. With this metric, and the equation of state given by Eq. (11), the energy-momentum conservation equation, $d\left(\rho_{W} R^{3}\right)=-p_{W} d\left(R^{3}\right)$, can be integrated to give

$$
\rho_{W}(R) \propto R^{-3(1+\alpha)}, \quad \alpha \equiv \frac{w^{2} \gamma^{2}-2}{3 \gamma^{2}} .
$$

The equation that governs cosmological evolution, $(\dot{R} / R)^{2}=(8 \pi G / 3) \rho_{W}$, can be integrated using Eq. (12) to give

$$
R(t) \sim t^{2 / 3(\alpha+1)}
$$




\section{ORIGINAL PAGE IS \\ OF POOR QUALITY}

The three cases of interest mentioned above will clearly correspond to the well-known solutions $R \sim t^{1 / 2}$ for $\alpha=1 / 3 ; R \sim t^{2}$ for $\alpha=-2 / 3$; and $R \sim t^{2 / 3}$ for $\alpha=0$.

Notice that for walls moving slowly enough, there may be a power-law inflation whenever the scale factor expands faster than $t$. It is easy to verify from Eq. (13) that the upper limit for the wall speed in order to have inflation is $w=1 / \sqrt{3}$, the speed of sound for a relativistic gas!

Before we move on to discuss the formation of walls, we would like to comment on the motion of walls in the presence of two fluids that may have (and do in most cases of interest) different "chemistry". In the case only the vacuum contributes to the energydensity of the Universe, the volume pressure accelerates the walls to $c$ ( $c$ is the velocity of light 'in vacuo'). The important point is that walls that are accelerated due to the difference in vacuum energy between the two sides may achieve a terminal velocity smaller than $c$ in case there is radiation or non-relativistic particles on both sides. As discussed in the work of Steinhart, ${ }^{9}$ this velocity is, however, larger than $1 / \sqrt{3}$ in most cases.

Steinhart ${ }^{9}$ has considered the motion of accelerated plane walls in the presence of two fluids, relativistic or not, at different temperatures on both sides of the wall. Only when the temperature of the fluid in the false vacuum is zero will the walls be accelerated to c. Using the language of "detonation waves", the wall can be understood as a wave front separating the burnt fluid, i.e. the true vacuum at the back of the wall, and the unburnt fluid, i.e. the false vacuum in front of the wall. The equivalent of the chemical energy stored in the unburnt fluid in a usual detonation is then the vacuum energy stored in the false vacuum. As the wave front propagates, it converts false vacuum into true vacuum, with the energy difference being used to further accelerate the wall and to heat up the fluid left behind. The wall drags with it the fluid just burnt, which will not be at rest with respect to the Universe, contrary to the thermalised fluid further away from the wall. Because the velocity of the wall with respect to the fluid immediately in front of it turns 
out to be larger than the speed of sound in the unburnt fluid, ${ }^{9}$ a point in the unburnt fluid cannot be perturbed by the wall until the wall passes by it. Thus, the unburnt fluid is at rest with respect to the cosmic rest frame.

Steinhart gives the velocity of the wall with respect to the unburnt fluid in contact with it, which is precisely the wall velocity $\omega$ that appeared in the equations above. The terminal velocity of the wall is in general larger or equal to $1 / \sqrt{3}$; this is the smaller possible velocity in the case that both fluids are relativistic. When the burnt fluid is relativistic and the unburnt fluid is non-relativistic the minimum terminal velocity is even larger, $\omega=\sqrt{3} / 2$. In the opposite case of a non-relativistic burnt fluid and a relativistic unburnt fluid the walls may move in the opposite direction, towards the true minimum side, if the vacuum energy cannot compensate the thermal pressure of the relativistic fluid in the false vacuum. This possibility has been raised in Ref. 8 , where it was shown that for reasonable values of the couplings the thermal pressure is dominated by the vacuum pressure, causing the wall to move towards the false vacuum. Recall also that as the Universe expands the thermal pressure becomes progressively weaker, with the walls eventually reaching relativistic speeds. 


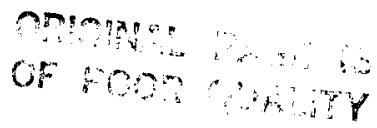

\section{Formation of Walls}

We now study the spontaneous breaking of an approximate discrete symmetry in the early Universe. First, some comments about the potential shown in Fig. 1. For the purpose of this paper, the exact way in which the asymmetry appears is immaterial, so long as the energy difference between the two vacua can be written as $\Lambda=\varepsilon \sigma_{0}^{4}$, where $\varepsilon$ can be a function of coupling constants and temperature. One example was given in Ref. 8 . In the limit $\varepsilon \rightarrow 0$ we recover the exact degeneracy of Eq. (1). The parameter $\varepsilon$ is the dimensionless asymmetry parameter.

Of course, the potential of Fig. 1 is the zero-temperature potential. It is only valid for temperatures safely below the critical temperature for the phase transition, $T_{c}$, which can be calculated for a particular model. For the hot big-bang model of the Universe it is reasonable to assume that temperatures higher than $T_{c}$ were achieved sufficiently early in the cosmological expansion. In this case one must use the temperature-corrected potential. Let us consider the $\varepsilon=0$ symmetric potential written in Eq. (1). For $T \gg T_{c}$ it is well known that the discrete symmetry will be restored and the potential will have a minimum at $\sigma=0$. The details of the phase transition for the symmetric case and the consequent formation of walls have been worked out by Kibble. ${ }^{5}$ Here we repeat some of his arguments so that we can compare them to the asymmetric case. Most of the results for an approximate symmetry have been derived in Ref. 8 .

First, it is not difficult to show that for the potential in Eq. (1) the critical temperature is $T_{c}=2 \sigma_{0}$. At zero temperature the two possible ground states for $\sigma$ are given by $\sigma=$ $\pm \sigma_{0}$. As the temperature approaches $T_{c}$, thermal fluctuations in the $\sigma$ field become large, with regions rapidly (compared to the cosmological time scale) interconverting between the two possible zero temperature values for $\sigma$. At high temperatures there is enough thermal energy in the system for the fluctuations to "jump over" the potential barrier. (At $T=0$, the height of the potential barrier is given by $U_{M}=(\lambda / 4) \sigma_{0}^{4}$.) The typical 
volume of a fluctuation region is given by $V_{\xi}=\xi^{3}$, where $\xi$ is the correlation length, given approximately by the inverse temperature-dependent mass of the $\sigma$ field, $\xi(T)^{-1}=$ $\lambda^{1 / 2} \sigma_{0}\left(1-T^{2} / T_{c}^{2}\right)^{1 / 2}$. At $T=0, \xi=\lambda^{-1 / 2} \sigma_{0}^{-1}$. Below $T_{c}$ the transition rate between the two vacua is proportional to $\exp \left(-F_{M} / T\right)$, where $F_{M}$ is the free energy of the fluctuation, $F_{M}=U_{M} \times V_{\xi}$. As the temperature decreases, the barrier between the two vacua increases while the thermal energy that drives the fluctuations in the $\sigma$ field decreases. One can define the Ginzburg temperature, $T_{G}$, as being the temperature below which fluctuations over the barrier will be exponentially suppressed and the population in the two vacua will be frozen. If one ignores the expansion of the Universe (in a more complete treatment one should obtain $T_{G}$ by comparing the thermal fluctuation rate, $\Gamma_{T}$, to the expansion rate of the Universe, $H: \Gamma_{T} \sim H$, at $T=T_{G}$ ), the Ginzburg temperature can be estimated to be $T_{G}=V_{\xi} U_{M} \cdot{ }^{5}$ In the case under consideration, $T_{G}=\sigma_{0}(\lambda+1 / 4)^{-1 / 2}$. Due to the perfect symmetry between the two vacua, the probability for a fluctuation to end up at $+\sigma_{0}$ or $-\sigma_{0}$ clearly is $50 \%$. Space will then be divided into cells of volume approximately given by $\xi^{3}$, with walls separating cells of positive and negative vacua. Of course, between cells of the same vacuum there will not be a domain wall. What will then be the general structure of space as the phase transition is completed? This question has been answered by studies of percolation in large lattices. ${ }^{10}$ It has been shown that if the probability for, say a plus-cell, is bigger than a certain value $p_{c}$, an infinite (in an infinite lattice) pluscluster appears, while if the probability is smaller than $p_{c}$ only finite plus-clusters appear. The value for $p_{c}$, the percolation threshold, varies with the type of lattice considered, but in all 3-dimensional lattices is smaller than $50 \%$. In the perfectly symmetric case both vacua have probabilities above $p_{c}$; space will be permeated by an infinite wall of very complicated topology dividing regions of plus and minus vacuum. There will also be small clusters but these will be exponentially suppressed.

The introduction of a small bias will change slightly the arguments above. For $T \gg T_{c}$ 
the potential will still have a parabolic shape but its minimum will not be exactly at $\sigma=0$. Its location will depend on the details of the model under consideration. Also, quantities such as $\mu, T_{c}, T_{G}$ and $\xi$ will have corrections proportional to the asymmetry parameter $\varepsilon$. However, we will assume that such corrections will not be very important due to the smallness of $\varepsilon$, and that it is a legitimate approximation to take for these quantities the same values as in the $\varepsilon=0$ case. The same cannot be said about the relative probability of having a fluctuation end up in a plus cell (denoted $p_{+}$) or in a minus cell (denoted $\left.p_{-}\right)$. The values for $p_{+}$and $p_{-}$are very sensitive to the energy difference between the two vacua, $\Lambda=\varepsilon \sigma_{0}^{4}$. As $\Lambda$ increases, the false vacuum, with larger free energy, becomes progressively more improbable. In fact, for $\varepsilon$ sufficiently large, the false vacuum may be below percolation threshold and only the true vacuum will percolate. This situation was analysed previously in the context of the formation of $\mathrm{NTSs}^{8}$ but will clearly not be of interest here; we must insure that both vacua percolate even though they will have different populations. This will effectively put an absolute upper bound on $\varepsilon$ which is obtained below.

As long as the system is in equilibrium, the relative population of the two vacua is given by the Boltzman formula, $p_{+} / p_{-}=\exp (-\Delta F / T)$, where $\Delta F=\Lambda \times V_{\xi}$ is the difference in free energy between the two minima. As explained above, these populations will be frozen as the temperature drops below $T_{G}$. Since $T_{G} \sim V_{\xi} \times U_{M}$, for $T<T_{G}$ we obtain,

$$
p_{+} / p_{-}=\exp \left(-\Lambda / U_{M}\right) \simeq \exp (-4 \varepsilon / \lambda)
$$

where the last result is obtained by taking $\Lambda \simeq \varepsilon \sigma_{0}^{4}$ and $U_{M} \simeq(\lambda / 4) \sigma_{0}^{4}$. Notice that we are using the expression for $U_{M}$ in the limit where $\varepsilon \rightarrow 0$. This approximation is valid as long as $U_{M} \gg \Lambda$, which is reasonable to expect for small asymmetry. We can now obtain the upper limit on $\varepsilon$ that results in percolation of both vacua. If one considers a simple cubic lattice (which we will, as a working model), $p_{c}=0.31 .^{10}$ Thus, solving Eq. (14) for 
$p_{+}>0.31$ one obtains

\section{ORIGINAL FAGE IO \\ OF POOR QUALITY}

$$
\varepsilon<0.2 \lambda \text {. }
$$

Other values for $p_{c}$ would just change slightly the numerical coefficient in front of $\lambda$ without changing the linear behaviour of $\varepsilon=\varepsilon(\lambda)$. With this upper value satisfied, even though the volume occupied by the true vacuum will be larger than that occupied by the false vacuum, at the completion of the phase transition space will be permeated by an infinite domain wall separating regions of plus and minus vacua.

We conclude this section by calculating the energy density in walls at formation, i.e., at $T=T_{G}$. In the last section we obtained the average energy density in walls to be $\rho_{W} \simeq \mu /\langle L\rangle$. We here give a more precise derivation. The wall energy density, $\rho_{W}$, is proportional to the surface tension $\mu$. The proportionality factor is just the ratio between the area in walls over the volume, $A_{\text {wall }} / V$. If we divide a space of volume $V$ into $N^{3}$ cubic cells of volume $\xi^{3}$ each, the probability of having a wall between two cells is given by twice the probability of having a plus-cell $p_{+}$followed by a minus-cell $1-p_{+}=p_{-}$, i.e. $2 p(1-p)$. Clearly, this is also the ratio between the area of the walls in the volume $V$ to the area of all cell-boundaries in $V, A_{\text {wall }} / A_{\text {total }}$. In order to obtain $A_{\text {wall }} / V$ we must calculate the ratio $A_{\text {total }} / V$, i.e. the ratio between the total area of cell boundaries over the volume. As we have $N^{3}$ cells of volume $\xi^{3}$ each, there will be $N-1$ internal boundaries between any two cells perpendicular to the $x$-axis, each with area $N^{2} \xi^{2}$, the same happening for the $y$ and $z$ axis. Thus, the total area in cell boundaries (i.e. boundaries between two cells, where walls may appear if the vacua on both sides are different) is $A_{\text {total }}=3(N-1) N^{2} \xi^{2} \simeq 3 N^{3} \xi^{2}$, where in the last expression the large $\mathbf{N}$ limit was assumed. The desired ratio is then $A_{\text {total }} / V=3 N^{3} \xi^{2} /(N \xi)^{3}=3 / \xi$. Thus,

$$
\frac{A_{\text {wall }}}{V}=\frac{A_{\text {wall }}}{A_{\text {total }}} \frac{A_{\text {total }}}{V}=\frac{3}{\xi} 2 p(1-p) .
$$

Using the value of $\mu$ obtained in Eq. (6), the approximate value for the correlation length 
at $T_{G}, \xi\left(T_{G}\right) \simeq\left(\lambda \sigma_{0}\right)^{-1}$, and Eq. (16), we find for the density in walls at formation

$$
\rho_{W}\left(T_{G}\right) \simeq 4 \sqrt{2} p(1-p) \lambda^{3 / 2} \sigma_{0}^{4}
$$

Thus, at $T_{G}$, the ratio of energy density in walls and radiation is [recall that $\rho_{R}=g_{*} T_{G}^{4} \simeq$ $g_{*} \sigma_{0}^{4}(\lambda+1 / 4)^{-2}$, where $g_{*}$ is the number of relativistic degrees of freedom at $T_{G}$ ],

$$
\left(\frac{\rho_{W}}{\rho_{R}}\right)_{T=T_{G}}=\frac{4 \sqrt{2}}{g_{*}} p(1-p) \lambda^{3 / 2}(\lambda+1 / 4)^{3 / 2} \simeq \frac{\lambda^{3 / 2}}{g_{*}} .
$$

As is well known (see Refs. 4-7, 11), the walls do not dominate the energy density of the Universe at formation.

\section{Dynamics of Walls I: Convoluted Structures Inside the Horizon}

As the walls are formed at $T_{G}$, space will be filled by the complicated wall structure described in the last section. Typically, the average wall separation $\langle L\rangle$, and the average curvature radius of the wall, $\bar{R}$, will be of the same order as the correlation length $\xi\left(T_{G}\right) \simeq$ $\left(\lambda \sigma_{0}\right)^{-1}$. The motion of the walls will be determined by two forces.

The first force is from the surface tension. Due to curvature the walls will feel a surface pressure that acts to straighten them. So long as the wall curvature is smaller than the horizon size, i.e., $\bar{R}<\lambda_{H} \simeq M_{P l} g_{*}^{-1 / 2} \sigma_{0}^{-2}$ where $M_{P l}$ is the Planck mass, this straightening will be rapid compared to the age of the Universe at that time. The surface pressure is given by $p_{T} \sim \mu / \bar{R}(t)$. At formation, $p_{T} \sim \lambda^{3 / 2} \sigma_{0}^{4}$. If this was the only force acting on walls, ${ }^{4}$ it would induce oscillations of frequency $\nu \sim \xi^{-1}$, quickly accelerating the walls to relativistic speeds. Of course, the motion of walls will be damped by radiation of particles and by the interactions of walls with each other and with the medium. ${ }^{5}$ As they evolve, the walls will become progressively flatter and slower up to the point where their curvature is of the same order as the horizon scale. Note that this damping mechanism is occuring on the scale of microphysics (we assume that $\sigma_{0} \ll M_{P l}$ ) 
and that the flattening of the walls will take a negligible time compared to the cosmological time scale. (For the readers convenience we include in Table 1 a complete list of all time scales used in this paper.) It is then easy to estimate the time when the walls can dominate the energy density of the Universe causing a power-law inflation (Refs. 4-6, 11), $\rho_{W}=\lambda^{1 / 2} \sigma_{0}^{3} / t>\rho_{R} \sim M_{P l}^{2} / t^{2}$ for $t>t_{\text {inf }} \simeq M_{P l}^{2} \lambda^{-1 / 2} \sigma_{0}^{-3}$. Let us call $t_{\text {inf }}$ the time at which power-law expansion starts, and $t_{G}$ the time of wall formation, for which $T=T_{G}$. Thus their ratio is simply $t_{\text {inf }} / t_{G} \simeq\left(g_{*}^{1 / 2} / \lambda^{1 / 2}\right)\left(M_{P l} / \sigma_{0}\right)$. This result was the root of the domain wall problem; the walls would eventually dominate the energy density of the Universe, causing a power-law inflation and conflict with astrophysical observations as explained in the introduction. Walls had to disappear before $t_{\text {inf }}$, or possibly some time not much larger than $t_{\text {inf }}{ }^{11}$ In the present scenario though, the walls will also suffer the influence of the volume pressure due to the asymmetry. The dynamics will be quite different.

The second force is a volume pressure on the walls due to the energy difference between the two vacua, $p_{V} \sim \varepsilon \sigma_{0}^{4}$. This pressure will accelerate the walls against the false vacuum regions, rapidly converting false vacuum into true vacuum. The energy released in the process fuels the wall motion. The process is very similar to the propagation of relativistic detonation waves analysed by Steinhardt. ${ }^{9}$ The wall will reach some terminal velocity $w_{\text {eq }}$ that will be dependent on the type of matter on both sides of the wall. (This is the wall velocity discussed in Section 2. The subscript eq was included now for clarity.) For example, the wall may be converting regions where a gauge symmetry is unbroken into a broken symmetry phase by giving mass to a certain particle, or the wall may be moving between relativistic fluids with different chemistry. The dynamics of the walls will be very sensitive to both the pressure and the tension forces; the exact treatment being out of the scope of this work.

The dynamics of the wall system will be examined in the various possible regimes that 
will depend upon when and which of the two forces dominate.

It is clear that the initial dynamics of the walls will depend on the balance between the two forces at formation. From the above, the ratio between the two pressures at $t=t_{G}$ is

$$
\left(\frac{p_{V}}{p_{T}}\right)_{t=t_{G}} \simeq \varepsilon \lambda^{-3 / 2}
$$

There will be two possible regimes. For $p_{V}>p_{T}$ (i.e., $\varepsilon>\lambda^{3 / 2}$ ), the volume pressure will dominate the motion of the walls from the moment they are formed; for $p_{V}<p_{T}$ (i.e., $\varepsilon<\lambda^{3 / 2}$ ), the tension will dominate the motion of the walls initially. Let us call these two regimes $A$ and $B$ respectively. Case $B$ can be further divided in two subcases, depending on whether the volume pressure acts on the walls before or after the tension has straightened them to the horizon scale. We call these two cases B.1 and B.2 respectively. Case B.2 is quite involved and will be treated separately in the next section. We proceed here to treat cases $\mathrm{A}$ and B.1, when the wall is still convoluted inside the horizon when $p_{V}$ dominates $p_{T}$. In Table 2 we present a brief summary of the four cosmological scenarios examined in this paper.

In addition to these conditions determining the initial behaviour of the walls, we must also ensure that both domains percolate by satisfying the inequality obtained in Eq. (15). (Otherwise the Universe would consist of a true vacuum sea with isolated bags of false vacuum that would, in the absence of stabilizing pressures, quickly disappear due to the tension force, as explained in Ref. 8.) In Fig. 2 (that should be interpreted together with Table 2), we show schematically the range for each of the two regimes with the percolation requirement satisfied. Notice that the two curves cross when $\lambda^{3 / 2}=0.2 \lambda$. The fact that case $\mathbf{A}$ is given by a lower bound for $\varepsilon$ imposes a strong constraint on the range of allowed parameter space; $\lambda<0.04$ in this case. The walls will be immediately accelerated against the false vacuum regions by the volume pressure. The acceleration is roughly, $a_{V} \sim p_{V} / \mu=\varepsilon \sigma_{0} / \lambda^{1 / 2}$. As the walls are separated initially by $\langle L\rangle \sim \xi\left(T_{G}\right)$, the 
ORIGINAL FAOE IS

OF. POOR QUALITY

time necessary for them to pinch off is $t_{\text {pinch }}^{-1} \sim\left(\lambda^{1 / 2} \varepsilon\right)^{1 / 2} \sigma_{0}$. They will disappear before any noticeable effect can occur on the cosmological time scale. For example, if $\lambda=10^{-2}$, $t_{\text {pinch }} / t_{G} \sim 10^{3} \sigma_{0} / M_{P l}$. There is no need to invoke Steinhardt's analysis for detonation waves because the time required for the shock front to acquire equilibrium, $t_{\mathrm{eq}} \sim w_{\mathrm{eq}} / a_{V}$, making his analysis applicable, is larger than $t_{\text {pinch }}$ by a factor of order $\varepsilon^{-1 / 2}$.

We can now move on to case B. The conditions on the asymmetry are $\varepsilon<\lambda^{3 / 2}$ and $\varepsilon<0.2 \lambda$. Thus, contrary to case $A$, only upper bounds are imposed on the asymmetry; as we will show, interesting scenarios are possible for small values of $\varepsilon$. In case B.1, the volume pressure becomes important before tension can straighten walls in a horizon scale but not immediately after formation, as in case A. As $p_{V}$ becomes dynamically effective, there will still be an entangled structure within a horizon size, although the average curvature radius and wall separation will now be bigger than at $t_{G}$ by an amount that scales with $\varepsilon^{-1}$. The condition that the volume pressure becomes effective before the walls are straight on a horizon scale imposes a lower bound for $\varepsilon: \varepsilon>\lambda^{1 / 2} \sigma_{0}^{-1} \bar{R}(t)^{-1}$. For $\bar{R}(t) \simeq \lambda_{H}$, we get

$$
\varepsilon>\left(\lambda g_{*}\right)^{1 / 2}\left(\frac{\sigma_{0}}{M_{P l}}\right)
$$

In Fig. 2 we show graphically the range of parameters that satisfy the three inequalities for case B.1. The motion of the walls is initially dominated by the tension force that will make them straight on scales up to $\bar{R}\left(t_{\varepsilon}\right)=\lambda^{1 / 2} / \varepsilon \sigma_{0}<\lambda_{H}$, when volume pressure dominates and starts accelerating walls toward the false vacuum regions. The disappearance of the walls in this case is very similar to case $A$, the difference being only on the scales. At $t_{\varepsilon}$ the walls will be separated on average by $\bar{R}\left(t_{\varepsilon}\right)$. The walls will disappear after a time $t_{\text {pinch }}^{-1} \simeq \lambda^{-1 / 2} \varepsilon \sigma_{0}$. Using (20) we obtain that $t_{\text {pinch }} \leq M_{P l} g_{*}^{-1 / 2} \sigma_{0}^{-2}=O\left(t_{G}\right)$, where $t_{G}$ is the age of the Universe at formation. So the pinching occurs before the walls can have any major role in the cosmological evolution. Steinhart's analysis is still not relevant, although now $t_{\text {eq }}$ is bigger than in case $A ; t_{\text {eq }} \sim \lambda^{1 / 2} / \varepsilon \sigma_{0} \simeq t_{\text {pinch }}$. The walls start pinching as 


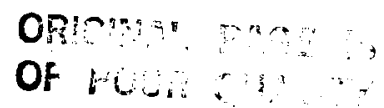

they reach terminal velocity, with the time scale for both processes being smaller than the cosmological time scale.

\section{Dynamics of Walls II: Structures Curved on a Horizon Scale}

In the last section we looked at the dynamics of walls in the case where the volume pressure coming from the asymmetry dominates the wall motion before the tension force can straighten the walls on a horizon scale. We have seen that the asymmetry causes the disappearance of the walls before they can play any major role in the cosmological evolution. In the present section we will consider the case when the asymmetry is sufficiently small in order to have walls with average separation and curvature comparable to the horizon size before the volume pressure starts to become effective.

This situation can still be subdivided into two cases (which are called cases B.2.1 and B.2.2 in Table 2 and in Fig. 2). One possibility, case B.2.1, is to have the volume pressure accelerating the walls before they can dominate the energy density of the Universe. This situation is the one explored earlier in the literature (see, e.g., Refs. 4-7). The other possibility, case B.2.2, is to have the walls dominating the energy density of the Universe before the volume pressure can act. Although the possibility of a wall-dominated Universe is perfectly reasonable, ${ }^{4,11}$ it is not clear to us what the evolution of such Universe will be like. We will present a rather naive approach to this case, being careful to point out the serious difficulties involved.

In order to have walls with curvature of the same order as the horizon we need simply to invert the inequality in Eq. (20),

$$
\varepsilon<\left(\lambda g_{*}\right)^{1 / 2}\left(\frac{\sigma_{0}}{M_{P l}}\right),
$$

with the other two conditions on $\varepsilon$ the same as in case B.1 (again, shown in Fig. 2). Once these three conditions are satisfied, the volume pressure will only become dynamically 
effective after the tension has straightened walls on scales up to $\lambda_{H}$. We still need to distinguish between the two possibilities discussed in the previous paragraph by imposing suitable bounds on $\varepsilon$. For this we simply compare $t_{\varepsilon}$, the time when the vacuum pressure dominates wall motion, to $t_{\text {inf }}$, the time when the walls dominate the energy density of the Universe. From the results in the previous section we get

$$
\frac{t_{\text {inf }}}{t_{\varepsilon}} \sim \frac{\varepsilon}{\lambda}\left(\frac{M_{P l}}{\sigma_{0}}\right)^{2}
$$

Thus, in order to avoid a wall-dominated Universe we must obey a lower bound for the asymmetry, $\varepsilon>\lambda\left(\sigma_{0} / M_{P l}\right)^{2}$. This lower bound has been found before by Vilenkin. ${ }^{6}$ It must, however, be supplemented by the other two upper bounds on $\varepsilon$ given in Eqs. (15) and (19) for this scenario to work. Assuming that we live in the true vacuum, the walls will be moving away towards the false vacuum with relativistic speeds that can be calculated using Steinhart's analysis. ${ }^{9}$ For us, it is important to note that the terminal velocity will be in most cases $1 / \sqrt{3} \leq w_{\text {eq }} \leq 1$, the exact value depending on the "chemistry" of the two phases. Of course, as the Universe expands and the temperature drops, wall velocities will asymptotically approach the speed of light. Such walls should not cause any cosmological damage.

The second possibility is to have the walls dominate the energy density of the Universe before the volume pressure turns on. From Eq. (22) we can see that this case obtains for exceedingly small asymmetry (unless, of course, $\sigma_{0} \sim M_{P l}$ ), that is, for almost degenerate vacua. As has been shown in Ref. 4 such Universe would have a power-law inflation with the scale factor evolving like $R(t) \sim t^{2}$. We have shown in Section 2 that this is indeed true in the limit of having a very non-relativistic gas of walls in a box. We question, however, if our analysis can be applied without contradictions to the situation with one wall per horizon volume. It seems that the more adequate approach to this situation would be to solve Einstein equations for one wall in a Robertson-Walker Universe. To the best of 
our knowledge, this has not been attempted so far. The possibility of a wall-dominated inflation remains an open question. Nevertheless, let us, for the sake of an argument, naively assume that wall-dominated inflation is possible once there is one wall per horizon and see what we can learn about the role of an approximate discrete symmetry in such Universe.

Assuming that the upper bound for $\epsilon<\lambda\left(\sigma_{0} / M_{P l}\right)^{2}$ is satisfied, the walls will be able to dominate the energy density of the Universe before the volume pressure turns on. Assuming further that the $t^{2}$ behavior for the scale factor is correct, we can ask how small $\varepsilon$ has to be in order to have sufficient inflation to solve the horizon and flatness problems of standard cosmology. ${ }^{12}$ In this naive picture, inflation would end once the walls are accelerated by the volume pressure to velocities larger than $w_{\text {eq }}=1 / \sqrt{3}$, since for $w_{\text {eq }} \geq 1 / \sqrt{3}$ the scale factor will evolve slower than the horizon, as shown in Section 2. As the walls are accelerated with $a_{V} \sim \varepsilon \sigma_{0} / \lambda^{1 / 2}$ it is easy to show that $t_{\text {endinf }}$, the time when inflation ends, and $t_{\varepsilon}$ are of the same order in comparison to the cosmological time scale. A rough estimate for the maximum (the exact calculation is quite involved since the exponent for the time evolution of the scale factor is itself a function of time) amount of inflation is then,

$$
\frac{R\left(t_{\text {endinf }}\right)}{R\left(t_{\text {inf }}\right)} \leq\left(\frac{t_{\text {endinf }}}{t_{\text {inf }}}\right)^{2} \sim \frac{\lambda^{2}}{\varepsilon^{2}}\left(\frac{\sigma_{0}}{M_{P l}}\right)^{4}
$$

In order to solve the horizon problem and make the walls disappear, we need $R\left(t_{\text {endinf }}\right) / R\left(t_{\text {inf }}\right) \geq 10^{26.12}$ Thus, the upper bound on $\varepsilon$ is

$$
\varepsilon \leq 10^{-13} \lambda\left(\frac{\sigma_{0}}{M_{P l}}\right)^{2} .
$$

A successful wall-dominated inflation would require, in this grossly simplified calculation, an extremely small asymmetry! Of course, even assuming that this analysis is qualitatively correct, only through simulations of wall motion including damping from the medium and the Hubble flow could a more explicit picture emerge. 


\section{Concluding Remarks}

The role of topological defects in cosmology was first studied in the work of Zel'dovich, Kobzarev and Okun. ${ }^{4}$ This work, and the extensive literature that it has inspired, has influenced the direction of model building in particle physics, in particular when discrete symmetries were involved, as for example in axion models and CP violation models. The dramatic results of Ref. 4, that particle theories with exact discrete symmetries are ruled out by cosmology, can be relaxed, as suggested in that very work, in the case the discrete symmetry is only approximate. As we hoped to have shown in this work, this is indeed the case.

We studied in some detail the cosmological consequences of having an approximate discrete symmetry spontaneously broken in the early Universe. We have shown that many possible scenarios are possible, depending on the value of the asymmetry parameter. Basically, after domain walls are formed in a primordial phase transition, their dynamics will be determined by the interplay between the tension force due to the curvature of the walls and the volume force coming from the volume energy stored in the false vacuum due to the asymmetry.

We divided the possible cases according to which of the two forces dominates first. Thus case $\mathbf{A}$ has the volume force dominant from the walls appearance while case $B$ has the tension force dominating the dynamics initially. In case $\mathrm{A}$ the walls disappear in a time much smaller than the cosmological time scale. Case B was divided into two subcases, depending on whether the tension force has acted to straighten the walls up to the scale of the horizon at the time when the volume pressure starts acting. In case B.1 the volume force acts before this can happen, and the walls disappear as they begin to be of any cosmological relevance. In case B.2, there will be essentially one wall per horizon before the volume pressure can act. This case can be further divided into two subcases, acrnrding to whether the energy density of the Universe is wall dominated or not. In the latter 


\section{ORIGINA? $P$ : \\ OF Fon an}

case, case B.2.1, the volume pressure will accelerate the wall to relativistic speeds and the Universe will be radiation dominated. In the first case, case B.2.2, if one accepts a naive approach to the problem of a wall-dominated Universe, we found that there can be an inflationary epoch that ends as the volume pressure starts accelerating the walls towards the false vacuum regions. Each of these scenarios obtains for certain bounds on the asymmetry that we evaluated in terms of the parameters involved in the simple model considered here.

\section{Acknowledgements}

We would like to thank A. Vilenkin for a useful conversation. This research was supported in part by the National Science Foundation under Grant No. PHY 82-17853, by DOE and by the National Aeronautics and Space Administration, at the University of California at Santa Barbara and at Fermi National Accelerator Laboratory.

\section{Table Captions}

Table 1. A time table for the different time scales defined in the text.

Table 2. A table of scenarios considered in the text. Recall that for formation of infinite walls, $\varepsilon<0.2 \lambda$ 


\section{ORIGINAL FAGE IS \\ OF POOR QUALITY \\ Figure Captions}

Fig 1. The zero temperature potential for non-degenerate vacua. $\varepsilon$, the asymmetry parameter, is in general function of temperature and of different couplings in the model.

Fig 2. Schematic display of all four possible scenarios for different values of the asymmetry parameter $\varepsilon$. In case $\mathbf{A}$ the walls disappear in a time scale much smaller than the cosmological time scale, $t_{G}$. In case B.1 the walls disappear in a time scale comparable to $t_{G}$. In case B.2.1 there can be one straight wall in a horizon volume but the walls disappear before dominating the energy density of the Universe. In case B.2.2 the walls dominate the energy density, causing a power law inflation until they disappear.

\section{References}

[1] For a review on domain walls and other topological defects see, A. Vilenkin,Phys. Rep. 121, 263 (1985).

[2] T. D. Lee, Phys. Rep. 9, (1974)

[3] R. D. Peccei and H. Quinn, Phys. Rev. Lett. 38, 1440 (1977); S. Weinberg, ibid. 40, 223 (1978); F. Wilczek, ibid. 40, 279 (1978); M. Dine, W. Fischler and M. Srednicki, Phys. Lett. 104B, 199 (1981); M. Wise, H. Georgi and S. L. Glashow, Phys. Rev. Lett. 47, $402(1981)$.

[4] Ya. B. Zel'dovich, I. Yu. Kobzarev and L. B. Okun, Zh. Eksp. Teor. Fiz. 67, 3 (1974) [Sov. Phys.-JETP 40, 1 (1975)].

[5] T. W. B. Kibble, J. Phys. A 9, 1387 (1976).

[6] A. Vilenkin, Phys. Rev. D23, 852 (1981).

[7] P. Sikivie, Phys. Rev. Lett. 48, 1156 (1982). 


\section{ORIGIMP: PHEE IS \\ OF POOR CUALITY}

[8] J. Frieman, G. Gelmini, M. Gleiser and E. Kolb, Phys. Rev. Lett. 60, 2101 (1988).

[9] P. J. Steinhart, Phys. Rev. D25, 2074 (1982).

[10] D. Stauffer, Phys. Rep. 54, 1 (1979).

[11] D. Seckel, in: “Inner Space/Outer Space”, eds. E. Kolb et. al. (Univ. of Chicago Press, Chicago, 1986).

[12] For a review on Inflation see, e.g., M. S. Turner, in: "Les Houches 1985", eds. P. Ramond and R. Stora (Elsevier Science Publishers B. V., 1987). 


\section{TABLE 1}

\begin{tabular}{|c|c|c|}
\hline Time scale & Definition & Time when... \\
\hline$t_{G}$ & $\simeq M_{\mathrm{Pl}} g_{*}^{-1 / 2} \sigma_{0}^{-2}$ & walls are formed. \\
\hline$t_{\text {inf }}$ & $\simeq M_{\mathrm{P} 1}^{2} \lambda^{-1 / 2} \sigma_{0}^{-3}$ & $\begin{array}{l}\text { walls dominate energy-density } \\
\text { of Universe. }\end{array}$ \\
\hline$t_{\text {pinch }}$ & $\simeq\left(\lambda^{1 / 2} \varepsilon\right)^{-1 / 2} \sigma_{0}^{-1}$ & walls pinch off. \\
\hline$t_{\text {eq }}$ & $\simeq w_{\mathrm{eq}} \lambda^{1 / 2}\left(\varepsilon \sigma_{0}\right)^{-1}$ & walls reach terminal velocity $w_{\text {eq. }}$ \\
\hline$t_{\varepsilon}$ & $\simeq \lambda^{1 / 2}\left(\varepsilon \sigma_{0}\right)^{-1}$ & vacuum pressure dominates wall motion. \\
\hline$t_{\text {endinf }}$ & $\simeq \lambda^{1 / 2}\left(\varepsilon \sigma_{0}\right)^{-1}$ & $\begin{array}{l}\text { wall dominated inflation ends } \\
\left.\text { (valid only for } \varepsilon<\lambda\left(\sigma_{0} / M_{\mathrm{Pl}}\right)^{2}\right)\end{array}$ \\
\hline
\end{tabular}




\section{TABLE 2}

\begin{tabular}{|c|c|c|}
\hline Case & Scenario & Bounds On Asymmetry \\
\hline $\mathbf{A}$ & $\begin{array}{l}\text { Volume pressure dominates } \\
\text { initially. Walls } \\
\text { disappear very fast } \\
\left(t_{\varepsilon} \ll t_{G}\right)\end{array}$ & $\varepsilon>\lambda^{3 / 2}$ \\
\hline B.1 & $\begin{array}{l}\text { Tension force dominates } \\
\text { initially but cannot straighten } \\
\text { walls up to horizon scale } \\
\left(t_{\varepsilon}<t_{G}\right)\end{array}$ & $\lambda^{3 / 2}>\varepsilon>\left(\lambda g_{*}\right)^{1 / 2}\left(\sigma_{0} / M_{\mathrm{P} 1}\right)$ \\
\hline B.2.1 & $\begin{array}{l}\text { Walls are curved on } \\
\text { a horizon scale but cannot } \\
\text { dominate energy-density } \\
\left(t_{\varepsilon}<t_{\text {inf }}\right) \text {. }\end{array}$ & $\begin{array}{l}\epsilon<\lambda^{3 / 2} ; \varepsilon<\left(\lambda g_{*}\right)^{1 / 2}\left(\sigma_{0} / M_{\mathrm{Pl}}\right) \\
\epsilon>\lambda\left(\sigma_{0} / M_{\mathrm{Pl}}\right)^{2}\end{array}$ \\
\hline B.2.2 & $\begin{array}{l}\text { Walls dominate energy-density } \\
\text { causing power law } \\
\text { inflation before disappearing } \\
\left(t_{\varepsilon}>t_{\text {inf }}\right) \text {. }\end{array}$ & $\begin{array}{l}\epsilon<\lambda^{3 / 2} ; \varepsilon<\left(\lambda g_{*}\right)^{1 / 2}\left(\sigma_{0} / M_{\mathrm{Pl}}\right) \\
\epsilon<\lambda\left(\sigma_{0} / M_{\mathrm{Pl}}\right)^{2}\end{array}$ \\
\hline
\end{tabular}


$\mathrm{U}(\sigma) / \lambda \sigma_{0}{ }^{4}$

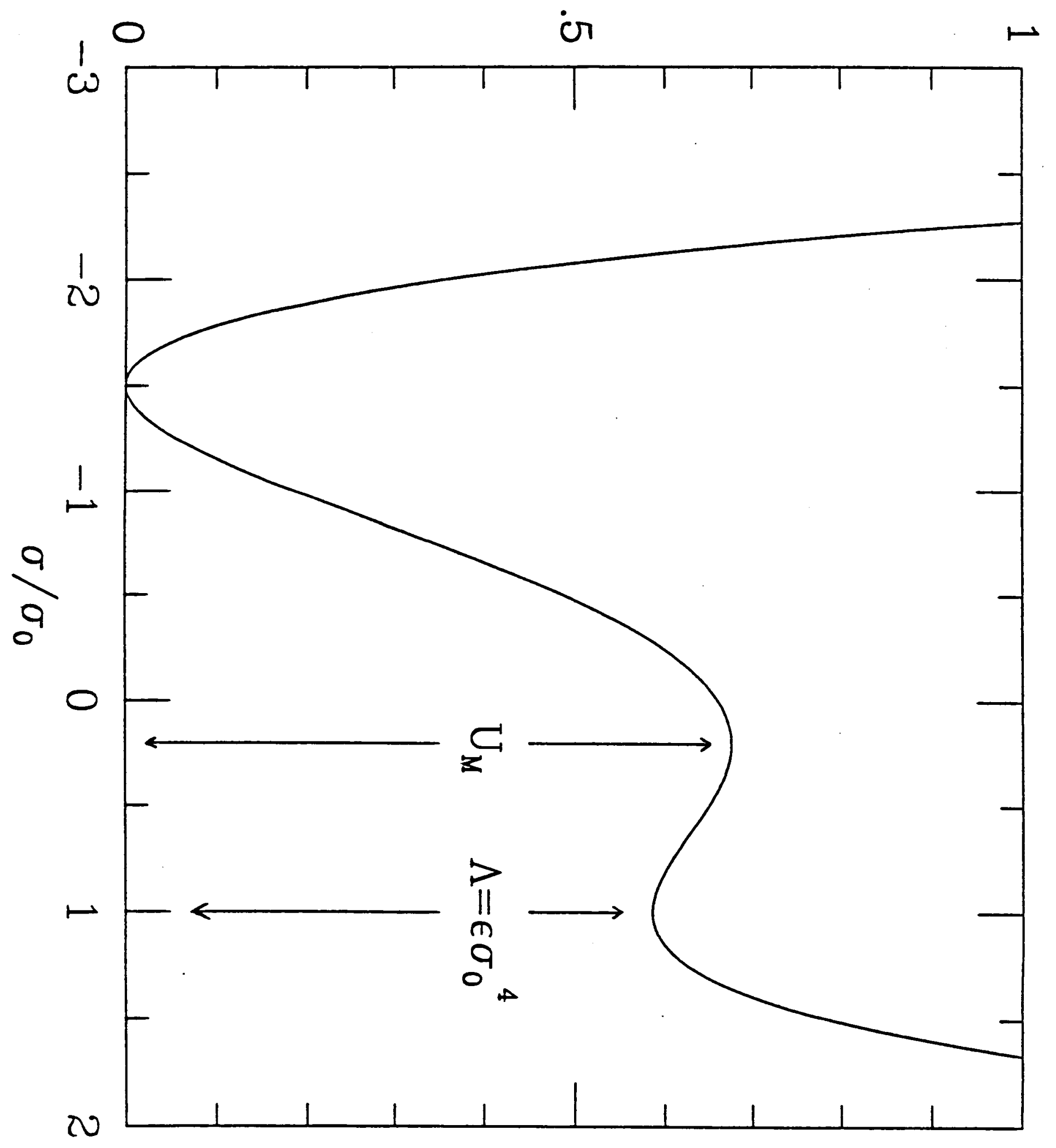

ALI7

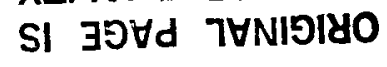


ORISINA PACE IS OF POOR QURITY

6.

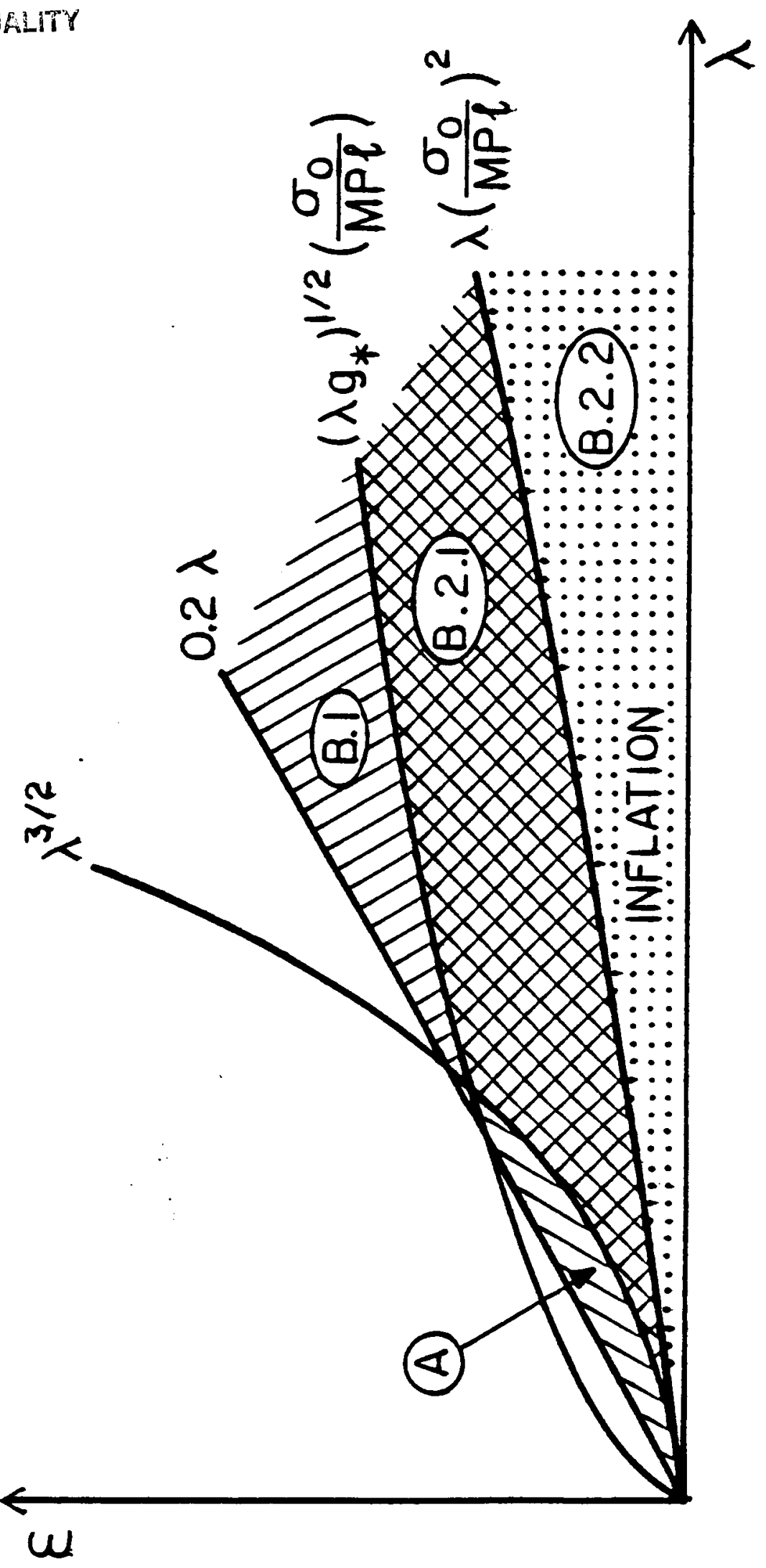

AII7t

Si 3OVd J NISIYO 